\title{
Observations on and Challenges to Research for the Future Treatments for Envenomation
}

\author{
Joseph A. Price* \\ Department of Pathology, OSU College of Osteopathic Medicine, Tulsa, Oklahoma, USA
}

${ }^{\star}$ Corresponding author: Joseph A. Price, Professor of Pathology, Department of Pathology, OSU College of Osteopathic Medicine, Tulsa, Oklahoma, USA; Phone: 918 561-1441; Email: joseph.price@okstate.edu

Received: January 29, 2019; Accepted: January 31, 2019; Published: February 04, 2019;

In this limited space I intend to make a few observations and opinions, and raise some questions, in order to stimulate thinking about future treatments for envenomation, mainly but not exclusively for the benefit of newer investigators and investigators new to this field. There is no way to be definitive completely in this short opinion piece. It seems fair to disclaim that while not all questions have simple answers, the process of consideration, including debate, stimulated in part by controversial statements and open questions, can lead to improved understanding, and hopefully better clinical outcomes.

Envenomation is a significant health challenge worldwide. Aside from medically serious hypersensitivities to venom peptides/ proteins from envenomation that would not otherwise be medically serious, e.g. by insects, the main concerns are snake and spider envenomation. The effects range from significant tissue loss to death. Traditional treatments have been the use of antisera in severe cases, and supportive care. The importance of treating envenomation and conducting research toward better treatment has periodically been specifically addressed by the World Health Organization.

Some time ago it was held in this country that you might acquire public research funding for a variety of projects, but not for a better understanding of the pathology of envenomation. The entrenched approach included reliance on antisera, and led to the Wyeth product for snake envenomation, helpful but with limitations for which it was difficult to impress many scientists that these existed and a better product was needed. Now it is no longer deemed safe and efficacious for human use, supplanted by a newer and more satisfactory product from abroad. Concerns limiting commercial development for many years, arguably with some validity, considered profitability of the development of such products. The recent involvement of at least one company in Mexico to develop products is another encouraging step, as has recent investment by the NIH, after a checkered history in this area. For neurotoxic snake venoms, one opinion expressed was that if the patient received timely supportive care in hospital, including respiratory support, they generally recover with minimal effects, without the use of anti-venom. Those are a lot of qualifications. One might ask what does real data say, both overall and for locales, and how uniformly is supportive care available.

We may also ask "Given the toll on populations in Africa and Asia, is the expenditure on research toward therapies for their envenomation at an appropriate level?", and if this is an economically disadvantaged area, "Is there a responsibility by the more economically advantaged countries to engage in meaningful contributions?" The less common the envenomation, and less profitable the market, the lower the likelihood of product development. "What is the value to humankind of the development of an effective, accessible treatment, that if found will be useful in the future in perpetuity?"

As has been often mentioned including in print, traditional treatments have had limitations. In the context of snake envenomation, hospitalization is not universal, antisera if locally available are expensive and perishable, and effectiveness varies. It is not always clear which species of animal was responsible, and the composition of venoms can vary substantially within a species, making the targeting of therapies a challenge.

Is there a single path of knowledge development, a single research approach to develop the new therapy, acceptable to the general body of researchers? I have had a reviewer indicate as much. But historically no; and for the future there seem developing options and pitfalls. A substantial volume of work has been to deconstruct venoms, with detailed in vitro enzymology, pharmacology and lately proteomics, studies of each toxin present, with much less of the venom's pathology in vivo. How comparable are studies done in vitro with reactions in simple buffers, occasionally with simple cell systems, compared to the clinically relevant in vivo complicated microenvironments of plasma and parenchymal tissue? For tissue destructive venoms, if a therapy to arrest tissue damage is found, can additional insights from wound management be applied to control excessive acute inflammation and hasten recovery, perhaps with tissue regeneration?

Lessons from and methods in drug development, from high throughput screening to lead optimization seem directly applicable. But to what extent are researchers trained in the study of purified enzymes in model reactions ready to consider the inhibition of the mixture of toxins, often enzymes in venoms, as is the actual clinical challenge, broadening their experimental systems, approaches and expectations, both in their own work and as grant or manuscript reviewers? Will established investigators adapt to new methodologies?

Is there a potential for enzyme inhibitors as a major component of anti-venom therapy, an idea that appears in print from the nineteen eighties? This idea has had a surge of interest recently, I hope in at 
least some small part due my own modest contributions, but recently by many others as well. Under what circumstances would the enzyme inhibitor approach have the best chances of success? Is the best target pathology due to venom with a single enzyme as the main virulence factor? Is the composition of venom across related species, within a species at different locales and developmental age of animal, sufficiently consistent a target for us to construct a simple yet effective cocktail of inhibitors, which if used in a single or brief dosing, will be effective but minimally toxic to the patient? Will it be possible to devise better, rapid, accurate diagnostic tests for the clinical lab to identify which venom is harming the patient, and point to specific therapies? The challenges are substantial, but equally so are the opportunities. 